\title{
Superhardness in boron carbide through nanostructuration
}

\author{
F. Igoa ${ }^{1,2}$, S. Delacroix ${ }^{1,2}$, Y. Song ${ }^{1}$, Y. Le Godec ${ }^{2}$, C. Coelho-Diogo ${ }^{3}$, C. Gervais ${ }^{1}$, G. Rousse ${ }^{4}$, D. Portehault ${ }^{1}$. \\ ${ }^{1}$ Sorbonne Université, CNRS, Laboratoire de Chimie de la Matière Condensée de Paris (LCMCP), Paris, France. \\ ${ }^{2}$ Sorbonne Université, CNRS, Institut de Minéralogie, Physique des Matériaux et Cosmochimie (IMPMC), Paris, France. \\ ${ }^{3}$ Sorbonne Université, CNRS, Institut des Matériaux de Paris Centre (IMPC), Paris, France. \\ ${ }^{4}$ Sorbonne Université, Collège de France, CNRS, Chimie du Solide et de l'Energie (CSE), Paris, France.
}

fernando.igoa_saldana@sorbonne-universite.fr

Production of nanostructures of extended covalent systems has remained a long-standing challenge, mainly due to the elevated activation energies required for their crystallization.[1] Such solids tend to exhibit outstanding mechanical properties, i.e. superhardness, the most illustrative case being diamond. Moreover, if nanostructuration is achieved (ideally in the $\approx 10 \mathrm{~nm}$ size range), further enhancement of the hardness can be obtained. For instance, diamond nanorods show an increase of the hardness by $86 \%$ compared to the bulk (from $80 \mathrm{GPa}$ to $150 \mathrm{GPa}$ ).[2] Superhard materials are of great industrial importance, with applications as cutting and polishing tools, coatings and abrasives. Diamond is indeed the traditional choice for such purposes, but it has well-known limitations: it is brittle, oxidizes to carbon dioxide at $800-900{ }^{\circ} \mathrm{C}$ in air and reacts with Fe-containing solids during cutting, not to mention the difficulty and cost of its production associated to the high-pressure machinery needed.

While several possible diamond substitutes have been suggested, boron carbide $\left(\mathrm{B}_{4+\delta} \mathrm{C}\right)$ stands as one of the few superhard phases that can be reached at room pressure. Boron carbide crystallizes in the $R \overline{3} m$ spacegroup and its network is based on B icosahedra linked to each other through both direct B-B bond and CBC chains, as depicted in Figure $1 . \mathrm{B}_{4+\delta} \mathrm{C}$ exhibits an intrinsic hardness of $38 \mathrm{GPa}$, yet far from the industrially profitable range. Plenty of effort has been devoted to the optimization of boron carbide's particle size and consequent amelioration of its mechanical properties. Approaches using different reactants, lower temperatures (down to $600^{\circ} \mathrm{C}$ ) and/or liquid-phase reactions have not been able to enable further lower the $\mathrm{B}_{4+\delta} \mathrm{C}$ particle size. In this work, instead of using pristine reagents, we demonstrate the capacity to produce $10 \mathrm{~nm} \mathrm{~B}{ }_{4+\delta} \mathrm{C}$ nanoparticles from a nano-precursor, namely $\mathrm{NaB}_{5} \mathrm{C}$. The structure of this cubic compound (space group $F d \overline{3} c$ ) resembles that of perovskites, where $\mathrm{B}_{5} \mathrm{C}$ octahedra form an anionic network that leaves cavities filled by $\mathrm{Na}^{+}$cations (Figure 1 left). $5-7 \mathrm{~nm} \mathrm{NaB}{ }_{5} \mathrm{C}$ nanoparticles were synthesized by using a high temperature liquid-phase procedure in molten salts.[3] The intrinsic carbon and boron mixture in a composition lying well within the range of the $\mathrm{B}_{4+\delta} \mathrm{C}$ solid solubility makes it an interesting precursor to yield boron carbide. Indeed, upon calcination, the $\mathrm{NaB}_{5} \mathrm{C}$ nanostructures are transformed to $\mathrm{B}_{4+\delta} \mathrm{C}$ with nanostructuration preservation at circa $10 \mathrm{~nm}$. After hot-pressing densification, the synthesized powders show enhancement of their mechanical properties above any previous record. We have used powder X-ray diffraction to shed light on the transformation from $\mathrm{NaB}_{5} \mathrm{C}$ to $\mathrm{B}_{4+\delta} \mathrm{C}$ at the atomic level. The implications of the new morphology of $\mathrm{B}_{4+\delta} \mathrm{C}$ on the mechanical properties will be discussed as well as the importance of the templating effect remaining from the original $\mathrm{NaB}_{5} \mathrm{C}$ nanostructures.
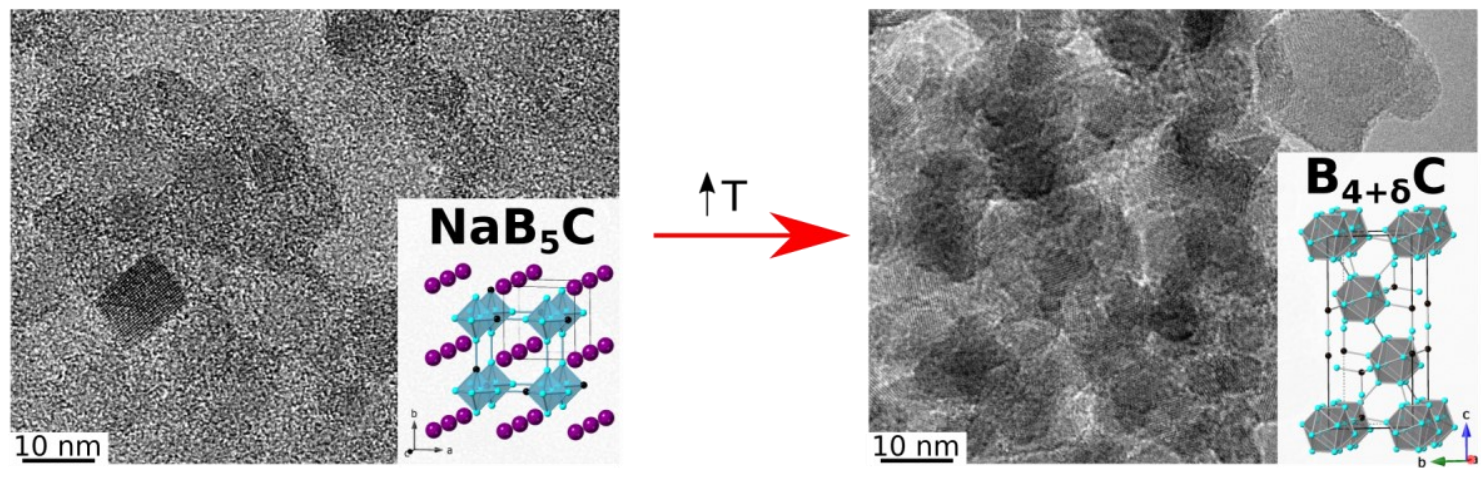

Figure 1. Transmission electron microscopy and crystal structures of recursor $\mathrm{NaB}_{5} \mathrm{C}$ (left) and product $\mathrm{B}_{4+\delta} \mathrm{C}$ (right).

[1] Solozhenko, V., et al. Adv. Mater. 2012, 24, 1540. [2] Irifune, T., et al. Nature. 2003, 421, 599. [3] Delacroix, S., Igoa, F., et al. Inorg. Chem. 2021, 60, 4252.

Keywords: Superhardness, Boron, Carbide, Ceramics, Phase transformation 